\title{
BOUNDARY CONDITIONS ON ARTIFICIAL FRONTIERS FOR INCOMPRESSIBLE AND COMPRESSIBLE NAVIER-STOKES EQUATIONS*
}

\author{
Charles-Henri Bruneau ${ }^{1}$
}

\begin{abstract}
Non reflecting boundary conditions on artificial frontiers of the domain are proposed for both incompressible and compressible Navier-Stokes equations. For incompressible flows, the boundary conditions lead to a well-posed problem, convey properly the vortices without any reflections on the artificial limits and allow to compute turbulent flows at high Reynolds numbers. For compressible flows, the boundary conditions convey properly the vortices without any reflections on the artificial limits and also avoid acoustic waves that go back into the flow and change its behaviour. Numerical tests illustrate the efficiency of the various boundary conditions.

Résumé. Les équations de Navier-Stokes pour des fluides incompressibles ou compressibles sont résolues avec des conditions aux limites non réfléchissantes. Dans le cas incompressible, les conditions aux limites conduisent à un problème bien posé, permettant de convecter correctement les tourbillons sans créer de réflexions et de faire des simulations directes de la turbulence à grands nombres de Reynolds. Dans le cas compressible, les conditions aux limites permettent aussi de convecter correctement les tourbillons et surtout d'éviter la remontée d'ondes acoustiques qui changent le comportement de l'écoulement. Des tests numériques illustrent l'efficacité des conditions aux limites proposées.
\end{abstract}

Mathematics Subject Classification. 35Q30, 65C20, 65M99.

Received: November 4, 1999.

\section{INTRODUCTION}

The aim of this work is very simple, we want to find the best conditions on the artificial limits of a computational domain, so that the computed solution on a small bounded domain will be close to the restriction of the solution computed on a larger one. The ideal case is reached when the computed solution on a bounded domain is equal to the restriction to that domain of the solution defined on an infinite domain. Many authors have already dealt with this problem and a lot of work has been done to derive relevant boundary conditions either directly in order to get a well-posed problem [17,21], or by using the theory of pseudo-differential operators to derive transparent or absorbing boundary conditions $[12,20]$. Nevertheless, the problem is not yet completely solved. Indeed, when dealing with compressible flows many computations use a buffer region technique (see $[11,23])$ to get a meaningful solution, as most of the boundary conditions create artificial acoustic waves that go back from the open limits into the flow field. These acoustic waves interact with the flow itself and can

\footnotetext{
Keywords and phrases. Navier-Stokes equations, artificial boundary conditions.

* Dedicated to Roger Temam for his 60th birthday

1 Mathématiques Appliquées de Bordeaux, Université Bordeaux 1, 351 cours de la Libération, 33405 Talence, France. e-mail: bruneau@math.u-bordeaux.fr
} 


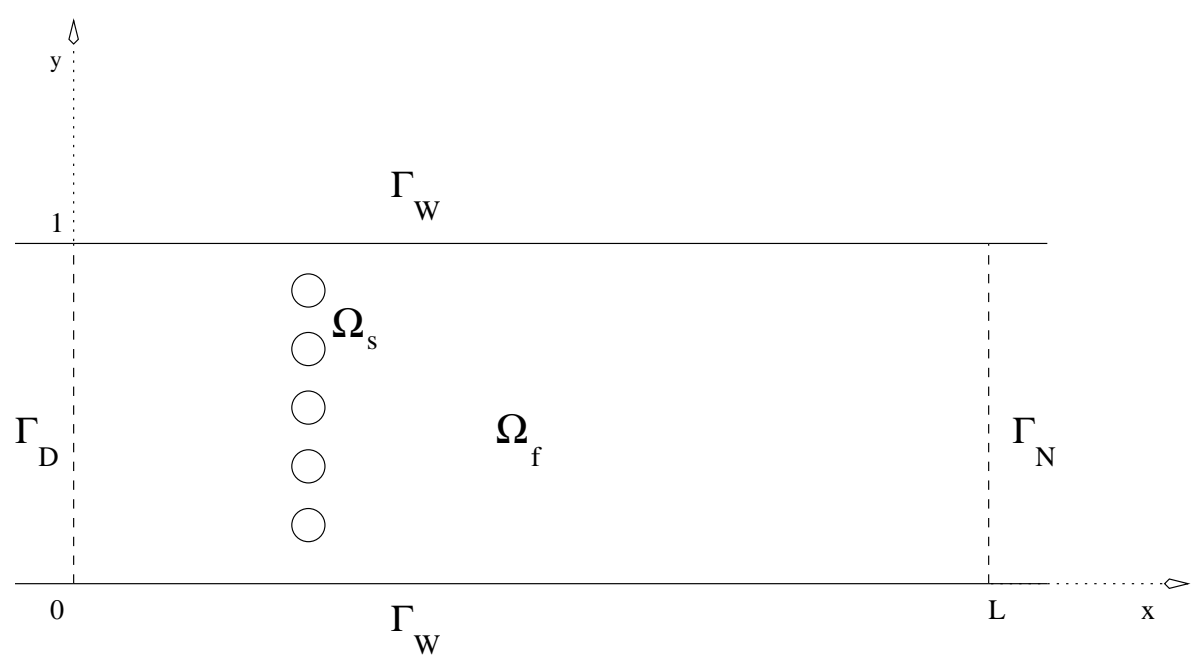

FIGURE 1. Numerical domain.

also reflect on the bodies to perturb the flow. Thus the behaviour is completely changed. For instance, artificial frequencies can be added to the main flow, changing a steady flow into an artificial periodic flow.

Here, we derive boundary conditions for incompressible fluids directly from a mixed formulation of NavierStokes equations keeping the positive part of the boundary term into the energy integral. The result is a condition that involves the stress tensor and a non linear term which occurs only when the flow is entering the domain at the artificial frontier of the domain. For compressible fluids, the characteristic waves for the corresponding Euler equations are used as in [19] to get a good evaluation of the waves entering the domain, assuming that the structures are convected by the main part of the flow. In both cases, a reference flow is needed and the key point is to use this reference flow to prescribe the right convection at the open boundary. Let us say for instance that in the incompressible case, the traction of the flow plus a non linear term are set equal to the traction of a reference flow. This reference flow can be either the flow at infinity or is derived from the computation.

This paper is divided into two parts. The first one is devoted to incompressible flows and is partly published in $[6,7]$. The second one concerns very new results on compressible flows [5]. In both cases, some non trivial numerical tests show the efficiency of the boundary conditions and their ability to convey properly strong phenomena in direct numerical simulations.

\section{OPEN BOUNDARY CONDITIONS FOR INCOMPRESSIBLE FLOWS}

\subsection{Preliminaries}

A discussion and analysis of the various forms of incompressible Navier-Stokes equations can be found for instance in $[13,18,22]$. Let $\Omega$ be a bounded domain in $\mathbb{R}^{2}$ or $\mathbb{R}^{3}$, we assume that $\Omega$ contains regular obstacles the reunion of which is called $\Omega_{s}$. We denote by $\Omega_{f}=\Omega \backslash \bar{\Omega}_{s}$ the domain for the incompressible fluid in which the Navier-Stokes equations are prescribed. The boundary of $\Omega_{f}$ is $\partial \Omega_{f}=\partial \Omega_{s} \cup \Gamma_{D} \cup \Gamma_{W} \cup \Gamma_{N}$ where $\Gamma_{D}$ corresponds to a part of the boundary (for instance an entrance section) where the flow is prescribed, $\Gamma_{W}$ denotes the solid walls and $\Gamma_{N}$ denotes the open boundaries (for instance an exit section) where a good boundary condition must be specified (see an example in Fig. 2). The motion is given by specifying a non homogeneous Dirichlet boundary condition on $\Gamma_{D}$ and a no-slip boundary condition is imposed on $\Gamma_{W}$. Then, the obstacles are taken into account by a penalization procedure which consists to add a penalized mass term in the equations which 
are now specified on the whole domain $\Omega[1,2]$. So, it is not necessary to specify a no-slip boundary condition on $\partial \Omega_{s}$. Finally, an open boundary condition is specified on $\Gamma_{N}$.

Thus, we are looking for the solution of the following initial boundary value problem:

$$
\begin{array}{rlrl}
\partial_{t} U+(U \cdot \nabla) U-\frac{1}{R e} \Delta U+\frac{1}{K} U+\nabla p & =F & & \text { in } \Omega_{T}=\Omega \times(0, T) \\
\operatorname{div} U & =0 & & \text { in } \Omega_{T} \\
U(X, 0) & =U_{0}(X) & \text { in } \Omega \\
U & =U_{D} & & \text { on } \Gamma_{D} \times(0, T) \\
U & =0 & & \text { on } \Gamma_{W} \times(0, T) \\
(U, p) \text { to be derived } & & & \text { on } \Gamma_{N} \times(0, T)
\end{array}
$$

where the vector $U$ is the velocity, $p$ the pressure, Re the Reynolds number, $U_{0}$ the initial datum and $U_{D}$ the flow at the entrance section. Note on one hand that the entrance must be far enough to the obstacles in order the Dirichlet condition on $\Gamma_{D}$ makes sense and on the other hand that we can have $\Gamma_{W}=\emptyset$. In this Navier-Stokes/Brinkman model, the scalar function $K$ can be considered as the permeability of the porous medium we are. Here we have the two opposites as the fluid should be of permeability infinite (set equal to $10^{16}$ ) and the solid of permeability zero (set equal to $10^{-8}$ ). Consequently, in the fluid the penalty term vanishes and the Navier-Stokes equations are solved whereas a Darcy equation is solved in the solid [1]. Then, the velocity vanishes in the obstacles. The computed values are of the same order of magnitude than $K$ inside $\Omega_{s}$. But, we get a continuous pressure field on the whole domain $\Omega$ that can be used to compute the drag and lift coefficients [9].

\subsection{The boundary condition}

The boundary condition can be seen as a natural Neumann-type boundary condition. Indeed, the first equation of (2.1) can be written in terms of the stress tensor $\left.\sigma(U, p)=\frac{1}{2 R e}\left(\nabla U+\nabla U^{t}\right)-p I\right)$ as:

$$
\partial_{t} U+(U \cdot \nabla) U-\operatorname{div} \sigma(U, p)+\frac{1}{K} U=F \quad \text { in } \quad \Omega_{T}
$$

Denoting $\left(U^{\text {ref }}, p^{\text {ref }}\right)$ the solution of the steady Stokes problem on $\Omega$ associated to the same data $U_{D}$ on $\Gamma_{D}$ and $F$, the variables $V=U-U^{\text {ref }}$ and $q=p-p^{\text {ref }}$ satisfy the first equation:

$$
\partial_{t} V+\left(\left(V+U^{\mathrm{ref}}\right) \cdot \nabla\right)\left(V+U^{\mathrm{ref}}\right)-\operatorname{div} \sigma(V, q)+\frac{1}{K}\left(V+U^{\mathrm{ref}}\right)=0 \text { in } \Omega_{T}
$$

and we get formally the weak formulation:

$$
\begin{array}{r}
\int_{\Omega} \partial_{t} V \cdot \Phi \mathrm{d} X+\int_{\Omega}\left(\left(V+U^{\mathrm{ref}}\right) \cdot \nabla\right) V \cdot \Phi \mathrm{d} X+\int_{\Omega}\left(\left(V+U^{\mathrm{ref}}\right) \cdot \nabla\right) U^{\mathrm{ref}} \cdot \Phi \mathrm{d} X-\int_{\Omega} \operatorname{div} \sigma(V, q) \cdot \Phi \mathrm{d} X \\
+\frac{1}{K} \int_{\Omega}\left(V+U^{\mathrm{ref}}\right) \cdot \Phi \mathrm{d} X=0
\end{array}
$$

where $\Phi$ is a regular test function which satisfies $\Phi=0$ on $\partial \Omega \backslash \Gamma_{N}$.

Then, we have by Green and Stokes formulas:

$$
\begin{array}{r}
\int_{\Omega} \partial_{t} V \cdot \Phi \mathrm{d} X+\frac{1}{2} \int_{\Omega}\left(\left(\left(V+U^{\mathrm{ref}}\right) \cdot \nabla\right) V \cdot \Phi-\left(\left(V+U^{\mathrm{ref}}\right) \cdot \nabla\right) \Phi \cdot V\right) \mathrm{d} X+\frac{1}{2} \int_{\Gamma_{N}}\left(V+U^{\mathrm{ref}}\right) \cdot n V \cdot \Phi \mathrm{d} \gamma \\
+\int_{\Omega}\left(\left(V+U^{\mathrm{ref}}\right) \cdot \nabla\right) U^{\mathrm{ref}} \cdot \Phi \mathrm{d} X+\int_{\Omega} \sigma(V, q): \nabla \Phi \mathrm{d} X-\int_{\Gamma_{N}} \sigma(V, q) \cdot n \cdot \Phi \mathrm{d} \gamma \\
+\frac{1}{K} \int_{\Omega}\left(V+U^{\mathrm{ref}}\right) \cdot \Phi \mathrm{d} X=0
\end{array}
$$


Setting the boundary terms in this last equation (2.5) to zero we can write down a Neumann-type boundary condition. But to take into account the local sign of the velocity on the artificial boundary, we can keep the positive part of $\left(\left(V+U^{\text {ref }}\right) \cdot n\right)$ in the weak formulation [7]. So, we can derive the boundary condition on $\Gamma_{N}$ for $(V, q)$ :

$$
\sigma(V, q) \cdot n+\frac{1}{2}\left(\left(V+U^{\mathrm{ref}}\right) \cdot n\right)^{-} V=0 \quad \text { on } \quad \Gamma_{N}
$$

Consequently the boundary value problem 2.1 has to be completed with the boundary condition for $(U, p)$ :

$$
\sigma(U, p) \cdot n+\frac{1}{2}(U \cdot n)^{-}\left(U-U^{\mathrm{ref}}\right)=\sigma\left(U^{\mathrm{ref}}, p^{\mathrm{ref}}\right) \cdot n \quad \text { on } \quad \Gamma_{N}
$$

For more details the reader should refer to [7] where a rigorous proof of the well-posedness of the boundary value problem $(2.1,2.7)$ is given. Let us remark that the non linear term is zero when $U \cdot n \geq 0$ which means that the flow is going out of the domain through $\Gamma_{N}$. On the contrary this term has to be taken into account when the flow is entering the domain. So, for Stokes flows the boundary condition (2.7) reduces to:

$$
\sigma(U, p) \cdot n=\sigma\left(U^{\mathrm{ref}}, p^{\mathrm{ref}}\right) \cdot n \quad \text { on } \quad \Gamma_{N}
$$

In order to use these boundary conditions we have to specify the reference flow $\left(U^{\text {ref }}, p^{\text {ref }}\right)$. To write down the equation (2.3), we have assumed that this flow is the solution of a steady Stokes problem with $U=U_{D}$ on $\Gamma_{D}$ which is in fact the flow at infinity. So, in many cases we can take for instance:

$$
U^{\text {ref }}=U_{\infty} \text { and } p^{\text {ref }}=p_{\infty}
$$

Nevertheless, such a reference flow does not give a good equivalent of the traction when the flow is chaotic or turbulent and consequently the condition becomes unstable for these regimes. A way to overcome this difficulty is to take as reference flow the flow computed just before the boundary at the same time step or at the previous one depending on the scheme used. Then, the reference flow changes at each time step and the boundary condition stay stable at high Reynolds numbers. But another difficulty can arise concerning the flowrate specially for internal flows. Indeed, in that case, the flowrate of the new reference flow can slightly deviate from the flowrate at the entrance section and time step after time step can induce a significant change corresponding to an external force applied to the exit section. So, it is necessary to control the flowrate of the reference flow.

\subsection{Numerical tests}

The numerical tests presented here concern the flow around a cylinder and the flow around obstacles (one or several cylinders) in a pipe. The numerical approximation stands on finite differences with a high-order scheme in time, implicit second-order differences for the pressure and diffusion terms, and explicit uncentered differences for convection terms. The resolution at each time step is achieved by means of a multigrid procedure in order to capture efficiently the whole range of the frequencies present in the flow. More details can be found in $[3,4]$. The boundary condition of the previous section has been implemented in $2 D$ and $3 D$ either for an open domain or for an exit section in a channel. The Reynolds numbers given until the end of the section are the real Reynolds numbers $R e=U_{\infty} l_{c} / \nu$ where the characteristic length $l_{c}$ is taken equal to the diameter of the cylinders. For the well-known test of the flow around a cylinder we find results in very good agreement with the physics [24] as it is shown in Table 1 where various values of the Strouhal number are compared. These tests were performed using as reference flow $\left(U^{\text {ref }}, p^{\text {ref }}\right)=\left(U_{\infty}, p_{\infty}\right)=\left((1,0)^{t}, 0\right)$ which is convenient for this kind of flow at low Reynolds numbers.

Now, we present some tests in a channel for various obstacles and Reynolds numbers in order to get interesting regimes. 
TABLE 1. Comparison of the Strouhal number around a cylinder.

\begin{tabular}{|c|c|c|}
\hline Reynolds number & Computed $S_{t}$ & Value of $S_{t}$ in $[24]$ \\
\hline 60 & 0.130 & 0.136 \\
\hline 100 & 0.164 & 0.164 \\
\hline 160 & 0.188 & 0.186 \\
\hline
\end{tabular}
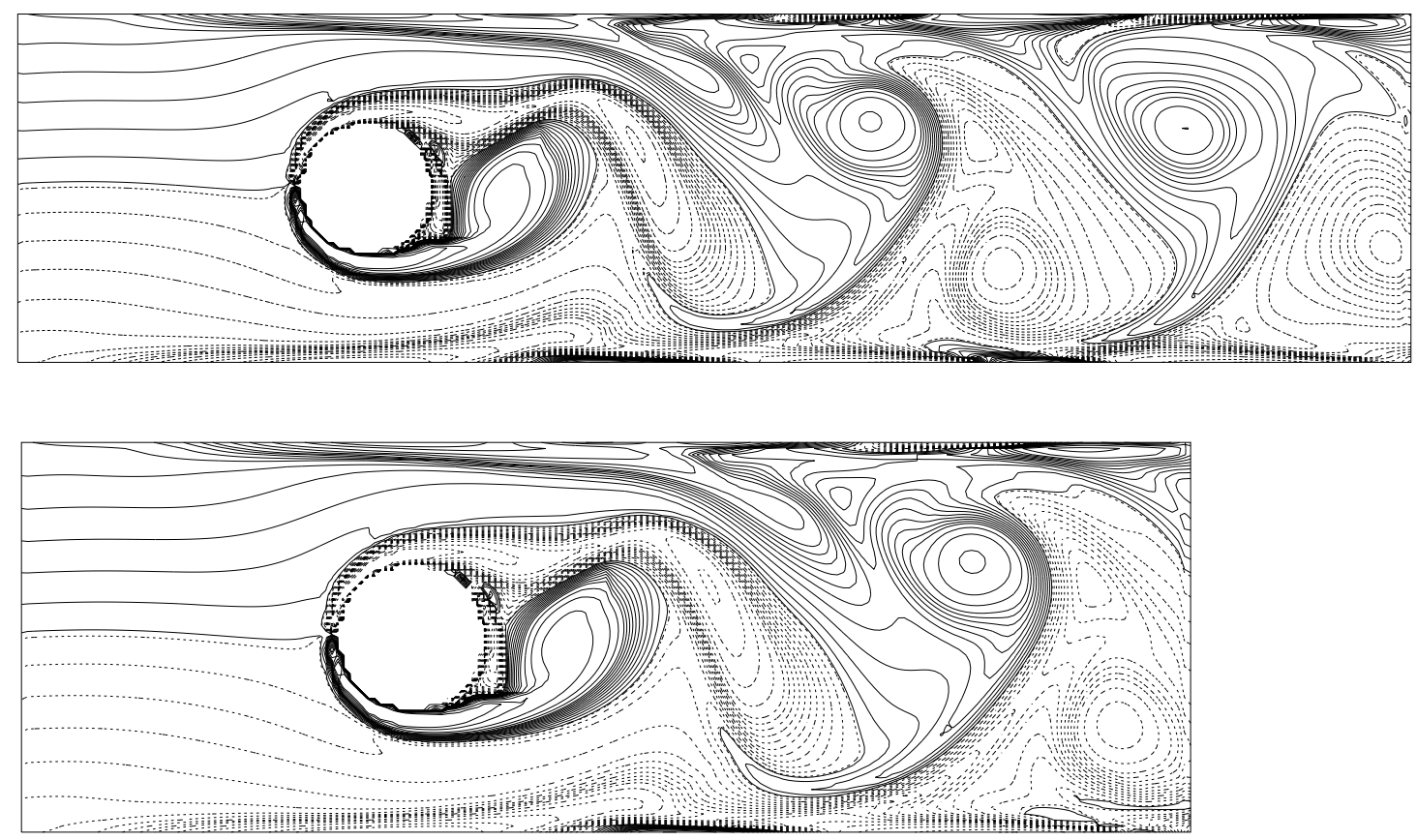

Figure 2. Comparison of the solutions obtained at $R e=400$ on the domain $\Omega$ with $L=4$ and $L=3$.

First of all, we compare the flow computed behind one single cylinder in a channel $\Omega$ with total length $L=4$ and $L=3$ to show the efficiency of the boundary condition. The flow at a medium Reynolds number $R e=400$ is periodic with strong alternate vortices convected to the artificial exit section of the infinite pipe. We can see in Figure 2 that the solution computed on the smaller domain is very close to the restriction of the solution computed on the larger one. We point out to the reader that both solutions are obtained after a long time of simulation corresponding to thirty periods of time and that the vortices are located at exactly the same place. In former works [6], we used Poiseuille flow as reference flow and got about the same solutions. This is true for low Reynolds numbers but not any more for high Reynolds numbers.

Now, we present the flow computed behind an array of five cylinders at very high Reynolds numbers on a regular mesh of $1920 \times 640$ cells. The array of cylinders is located at a length of 1 from the entrance section and the total length of the domain is $L=3$. The diameter of the cylinders is 0.1 and the spacing between two centers of cylinders is 0.2 . The vorticity contours are displayed in Figure 3 for a simulation at $R e=5 \times 10^{5}$ at a time such that the full regime is well established. The numerical simulation at such a high Reynolds number is made possible by the choice of the computed flow before the exit section as reference flow. Indeed, with the choice of Poiseuille flow it is not possible to get the solution when $R e>5 \times 10^{3}$. In Figure 3, we see that the 


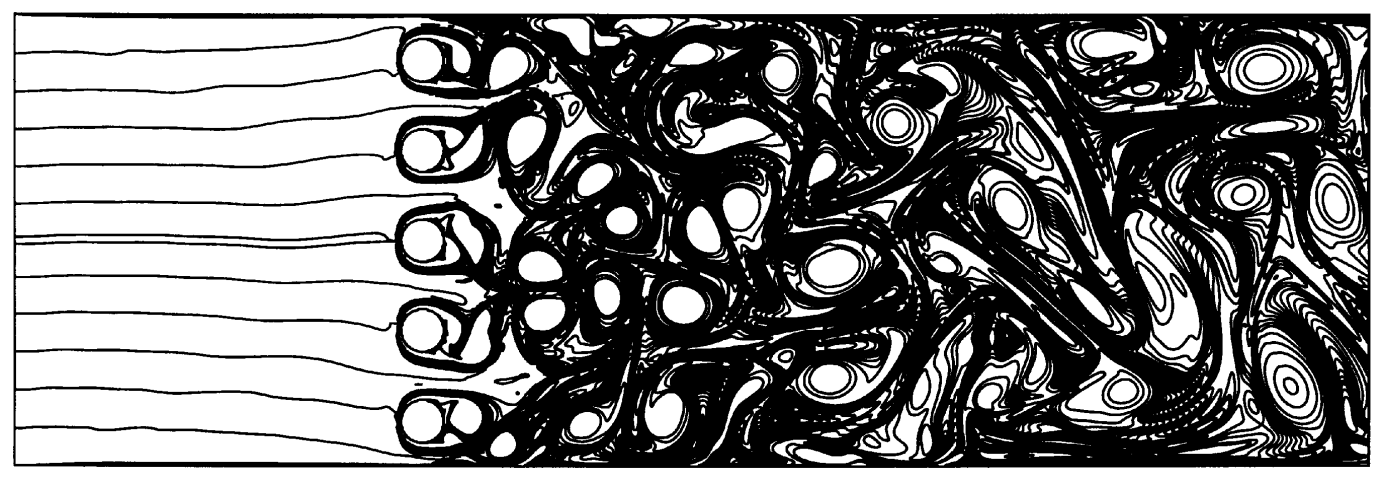

FiguRE 3. Vorticity contours at $R e=5 \times 10^{5}$.

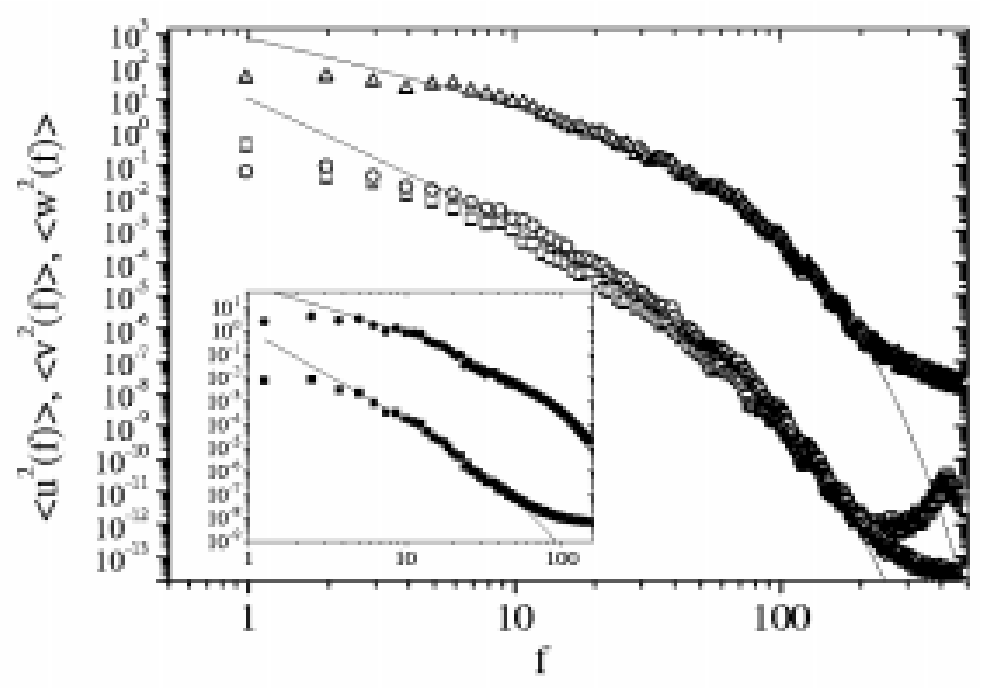

FiguRE 4. Spectra.

flow before the cylinders is laminar and displays a Poiseuille profile with mean velocity equal to 1 . After the passage by the grid, the flow is dominated by the presence of vortices interacting very strongly with each other. The strong vorticity gradients are well captured and correspond to the dark filaments on the plot. The size of the vortices which is close to the diameter of the cylinders just behind them grows as they are convected downstream. This coarsening of the coherent structures is due to the vortex mergers typical of $2 D$ turbulence.

To better analyse the turbulent flow, a time trace is recorded at different points randomly chosen in the flow. This is done for the two components of the velocity $U=(u, v)^{t}$, the pressure $p$ and the vorticity $\omega$. The spectra displayed in Figure 4 correspond to one point located at about one channel width down from the array of cylinders. The spectra for the two components of the velocity (squares for $u$ and circles for $v$ ) have roughly the same amplitude in the frequency range studied, showing that the turbulence is nearly isotropic. Both spectra show a small flat part at low frequencies and a continuous decrease as the frequency is increased which becomes steeper for high frequencies. The whole spectra can be fit to the analytical function $f^{-\beta} e^{-f / f_{0}}$ where the cutoff frequency $f_{0}=18$ and the exponent $\beta=3.7$. The same observations are valid for the enstrophy spectrum (triangles) except the exponent $\beta=1.9$. The inset of Figure 4 shows the same features for the energy 


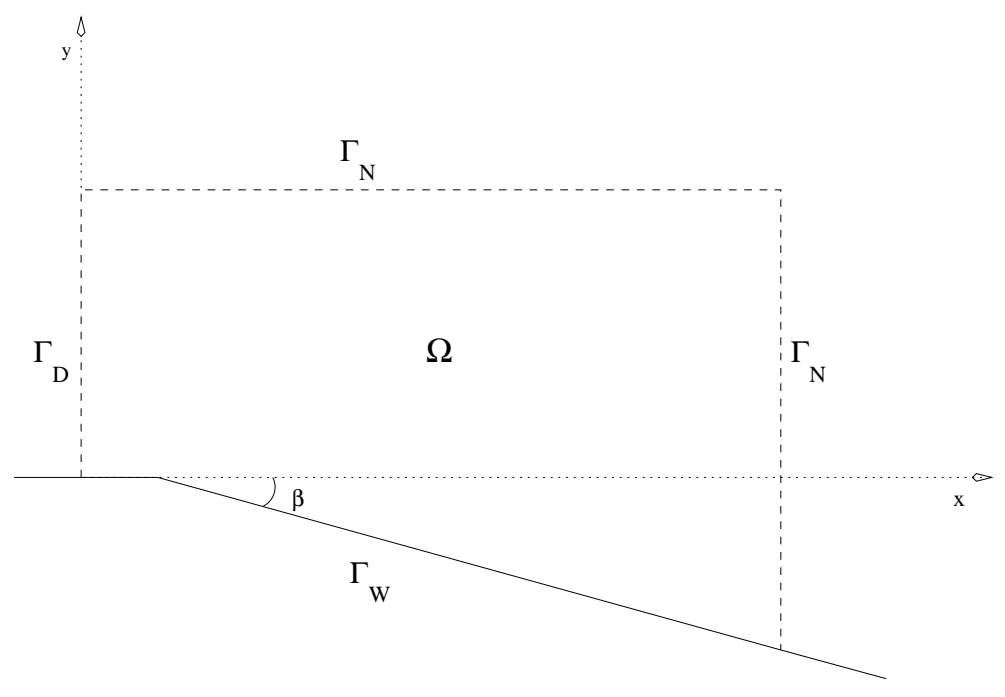

FIGURE 5. Numerical domain.

density (squares) and the enstrophy (circles) spectra obtained from $2 D$ Fourier transforms of the $2 D$ velocity and vorticity fields.

This direct numerical simulation is also compared to an experiment. This experiment is carried out in a vertical soap film formed between two parallel thin wires, as described in details in [15]. The film falls under the action of gravity at speeds controlled by the injection flux. The turbulence in the film is generated by a comb that is thrust perpendicularly through the film. The interaction between the wakes coming from the different cylinders produces nearly isotropic turbulence some distance behind the grid. Although the results seem to be consistent with expectations for two-dimensional decaying turbulence [10], the vorticity results indicate deviations from the theoretical predictions [16]. A good agreement between numerical simulations and the experiments is obtained for energy and enstrophy spectra as well as for the moments of longitudinal velocity differences and the probability density functions of the enstrophy flux. More details can be found in $[8,14]$.

\section{OPEN BOUNDARY CONDITIONS FOR COMPRESSIBLE FLOWS}

\subsection{Preliminaries}

In this section, we consider a domain $\Omega$ without obstacles with boundary $\partial \Omega=\Gamma_{D} \cup \Gamma_{W} \cup \Gamma_{N}$ where, as in the previous section, $\Gamma_{D}$ corresponds to a part of the boundary where the flow is prescribed, $\Gamma_{W}$ denotes the solid walls and $\Gamma_{N}$ denotes the open boundaries where a good boundary condition must be specified to avoid reflections. (see an example in Fig. 5). Eventually, the part $\Gamma_{W}$ can be empty to test the convection of a vortex through an artificial limit.

On this domain $\Omega$ the $2 \mathrm{D}$ compressible Navier-Stokes equations for the density $\rho$, the velocity $V=(u, v)^{t}$ and the total energy $e$ are prescribed:

$$
\left\{\begin{array}{llll}
\frac{\partial U}{\partial t}+\frac{\partial F_{x}(U)}{\partial x}+\frac{\partial F_{y}(U)}{\partial y} & =\frac{1}{R e}\left(\frac{\partial G_{x}(U, \nabla U)}{\partial x}+\frac{\partial G_{y}(U, \nabla U)}{\partial y}\right) & \text { in } & \Omega_{T} \\
U(x, 0) & =U_{0}(x) & \text { in } \Omega \\
U & =U_{D} & \text { on } \Gamma_{D} \times(0, T) \\
U & =0 & \text { on } \Gamma_{W} \times(0, T) \\
U \text { to be derived } & & \text { on } \Gamma_{N} \times(0, T)
\end{array}\right.
$$


with:

$$
\begin{gathered}
U=\left[\begin{array}{c}
\rho \\
\rho u \\
\rho v \\
\rho e
\end{array}\right] \quad F_{x}(U)=\left[\begin{array}{l}
\rho u \\
\rho u^{2}+p \\
\rho u v \\
(\rho e+p) u
\end{array}\right] \quad F_{y}(U)=\left[\begin{array}{l}
\rho v \\
\rho u v \\
\rho v^{2}+p \\
(\rho e+p) v
\end{array}\right] \\
G_{x}(U, \nabla U)=\left[\begin{array}{l}
0 \\
\sigma_{x x} \\
\sigma_{x y} \\
\beta_{x}
\end{array}\right] \quad G_{y}(U, \nabla U)=\left[\begin{array}{l}
0 \\
\sigma_{x y} \\
\sigma_{y y} \\
\beta_{y}
\end{array}\right]
\end{gathered}
$$

where:

$$
\left\{\begin{array} { r l } 
{ \sigma _ { x x } } & { = \frac { 4 } { 3 } \frac { \partial u } { \partial x } - \frac { 2 } { 3 } \frac { \partial v } { \partial y } } \\
{ \sigma _ { y y } } & { = \frac { 4 } { 3 } \frac { \partial v } { \partial y } - \frac { 2 } { 3 } \frac { \partial u } { \partial x } } \\
{ \sigma _ { x y } } & { = \frac { \partial u } { \partial y } + \frac { \partial v } { \partial x } }
\end{array} \quad \left\{\begin{array}{l}
\beta_{x}=u \sigma_{x x}+v \sigma_{x y}+\frac{\gamma k}{\operatorname{Pr}} \frac{\partial \Theta}{\partial x} \\
\beta_{y}=u \sigma_{x y}+v \sigma_{y y}+\frac{\gamma k}{\operatorname{Pr}} \frac{\partial \Theta}{\partial y}
\end{array}\right.\right.
$$

$R e$ is the Reynolds number with the same definition as above where $l_{c}$ depends on the test problem, $\gamma, k$ and $\operatorname{Pr}$ are respectively the perfect gaz constant, the thermal conductivity coefficient and the Prandtl number which is constant here. To eliminate the pressure $p$ in these equations, we add the state equation $p=(\gamma-1) \rho \Theta$. When the flow is supersonic it is not necessary to prescribe something on the artificial frontiers $\Gamma_{N}$ of the domain as all the characteristics curves are leaving the domain $\Omega$. Here, we are interesting in subsonic flows for which one or several quantities depending on the sign of the local velocity have to be derived.

\subsection{The boundary condition}

To discretize the equations we use a finite volumes-finite elements approximation of the conservative variables except on the boundary where the normal derivative of the convective flux is derived from the characteristic variables. So, the boundary condition is derived from the discretization of the characteristic waves amplitude vector $L$ of the unviscous part of the equations (3.1) which is given on $\Gamma_{N}$ with outside unit normal $n=(1,0)^{t}$ by (see [19] or [5]):

$$
L=\left[\begin{array}{c}
L_{1} \\
L_{2} \\
L_{3} \\
L_{4}
\end{array}\right]=\left[\begin{array}{c}
(u-c)\left(\frac{\partial p}{\partial x}-\rho c \frac{\partial u}{\partial x}\right) \\
u\left(c^{2} \frac{\partial \rho}{\partial x}-\frac{\partial p}{\partial x}\right) \\
u\left(\frac{\partial v}{\partial x}\right) \\
(u+c)\left(\frac{\partial p}{\partial x}+\rho c \frac{\partial u}{\partial x}\right)
\end{array}\right]=\left[\begin{array}{c}
\lambda_{1}\left(\frac{\partial p}{\partial x}-\rho c \frac{\partial u}{\partial x}\right) \\
\lambda_{2}\left(c^{2} \frac{\partial \rho}{\partial x}-\frac{\partial p}{\partial x}\right) \\
\lambda_{3}\left(\frac{\partial v}{\partial x}\right) \\
\lambda_{4}\left(\frac{\partial p}{\partial x}+\rho c \frac{\partial u}{\partial x}\right)
\end{array}\right]
$$

where $c$ is the local speed of sound and $\lambda_{k} 1 \leq k \leq 4$ are the eigenvalues of the Euler operator. If the flow is subsonic and $u \geq 0$, the first characteristic curve is entering the domain and thus $L_{1}$ must be evaluated from outside the domain. If $u<0$, then it is the same for $L_{2}$ and $L_{3}$. In [19], $L_{1}$ is either set equal to zero or to a function of $p-p_{\infty}$ in order to introduce the pressure at infinity as $p=p_{\infty}$ is a relevant boundary condition for subsonic flows. Here, we propose to discretize $L_{1}$ from outside the domain according to the way of propagation 
of that wave using a steady reference flow $U^{\text {ref }}$ which is supposed to represent properly the main part of the flow. In several cases the reference flow can be taken equal to the flow at infinity or derived from it. In some cases like for the flow over a dihedral plate (Fig. 5) it is necessary to compute a steady solution at low Reynolds number to get the reference flow. Then, we write down $U=U^{\text {ref }}+U^{\prime}$ where $U^{\prime}$ is the deviation from the chosen reference flow. Now, if for the sake of simplicity we consider a Cartesian uniform mesh around one point $P_{i, j}$ on the frontier $\Gamma_{N}$, we can write at time $n \delta t$ for the first component:

$$
\begin{aligned}
\left(L_{1}\right)_{i, j}^{n}= & \left(u_{i, j}^{n}-c_{i, j}^{n}\right)\left(\frac{-p_{i+2, j}^{\mathrm{ref}}+4 p_{i+1, j}^{\mathrm{ref}}-3 p_{i, j}^{\mathrm{ref}}}{2 \delta x}-\rho_{i, j}^{n} c_{i, j}^{n} \frac{-u_{i+2, j}^{\mathrm{ref}}+4 u_{i+1, j}^{\mathrm{ref}}-3 u_{i, j}^{\mathrm{ref}}}{2 \delta x}\right) \\
& +\left(u_{i, j}^{n}-c_{i, j}^{n}\right)\left(\frac{-\left(p^{\prime}\right)_{i+2, j}^{n}+4\left(p^{\prime}\right)_{i+1, j}^{n}-3\left(p^{\prime}\right)_{i, j}^{n}}{2 \delta x}-\rho_{i, j}^{n} c_{i, j}^{n} \frac{-\left(u^{\prime}\right)_{i+2, j}^{n}+4\left(u^{\prime}\right)_{i+1, j}^{n}-3\left(u^{\prime}\right)_{i, j}^{n}}{2 \delta x}\right) \\
= & \left(L_{1}^{\mathrm{ref}}\right)_{i, j}^{n}+\left(L_{1}^{\prime}\right)_{i, j}^{n}
\end{aligned}
$$

the first part of which is computed with the reference flow and the second part is approximated in time as follow:

$$
\begin{aligned}
\left(L_{1}^{\prime}\right)_{i, j}^{n} & \approx\left(u_{i, j}^{n}-c_{i, j}^{n}\right)\left(\frac{-\left(p^{\prime}\right)_{i, j}^{n-2 \alpha}+4\left(p^{\prime}\right)_{i, j}^{n-\alpha}-3\left(p^{\prime}\right)_{i, j}^{n}}{2 \delta x}-\rho_{i, j}^{n} c_{i, j}^{n} \frac{-\left(u^{\prime}\right)_{i, j}^{n-2 \alpha}+4\left(u^{\prime}\right)_{i, j}^{n-\alpha}-3\left(u^{\prime}\right)_{i, j}^{n}}{2 \delta x}\right) \\
& =\left(u_{i, j}^{n}-c_{i, j}^{n}\right)\left(\frac{-p_{i, j}^{n-2 \alpha}+4 p_{i, j}^{n-\alpha}-3 p_{i, j}^{n}}{2 \delta x}-\rho_{i, j}^{n} c_{i, j}^{n} \frac{-u_{i, j}^{n-2 \alpha}+4 u_{i, j}^{n-\alpha}-3 u_{i, j}^{n}}{2 \delta x}\right)
\end{aligned}
$$

as the reference flow is stationary. In equation (3.4) we define $t_{n-\alpha}=t_{n}-\alpha \delta t$ where $\alpha=q_{\max } / V_{\text {conv }}$ with $q_{\max }$ the modulus of the maximum velocity used in the CFL condition and $V_{\text {conv }}$ the convection velocity on $\Gamma_{N}$. Indeed, we assume that the value $\left(p^{\prime}\right)_{i+1, j}^{n}$ outside of the domain can be replaced by $\left(p^{\prime}\right)_{i, j}^{n-\alpha}$ on the frontier of the domain at a previous time which corresponds to the time necessary to convect the phenomena from point $P_{i, j}$ to point $P_{i+1, j}$. In some applications we can have:

$$
V_{\text {conv }}=V^{\mathrm{ref}}=V_{\infty}
$$

But in some situations it is necessary to take $V_{\text {conv }} \neq V^{\text {ref }}$ as $V^{\text {ref }}$ can be very small. For instance in the neighbourhood of the boundary layer $V^{\text {ref }}$ is very small but the phenomena are convected by the mean flow. So, taking $V_{\text {conv }}$ instead of $V^{\text {ref }}$ is better as it involves shorter times in the past.

\subsection{Numerical tests}

The first test presented here concern the convection of an inviscid vortex by an uniform constant flow. At the initial time, the vortex is located in the middle of a square domain. Then, it is convected from left to right by an uniform velocity $V_{\infty}=\left(u_{\infty}, v_{\infty}\right)^{t}=(1,0)^{t}$ and we compare the solution obtained with the non-reflecting boundary condition (NRBC) presented in [19] and the one obtained with the present boundary condition (PBC) to a reference solution computed on a large domain, so that the vortex does not interact with the artificial frontier. To apply our boundary condition we have taken $V^{\text {ref }}$ and $V_{\text {conv }}$ equal to $V_{\infty}$ as proposed above (3.5).

As can be seen in Figure 6, the PBC solution is quite identical to the reference solution whereas the NRBC solution has a vorticity field slightly deformed and provides strong acoustic waves that go back into the flow field. In addition, the vorticity evacuation is very good and the reflection coefficient is very low with the PBC as it is detailed in [5]. 

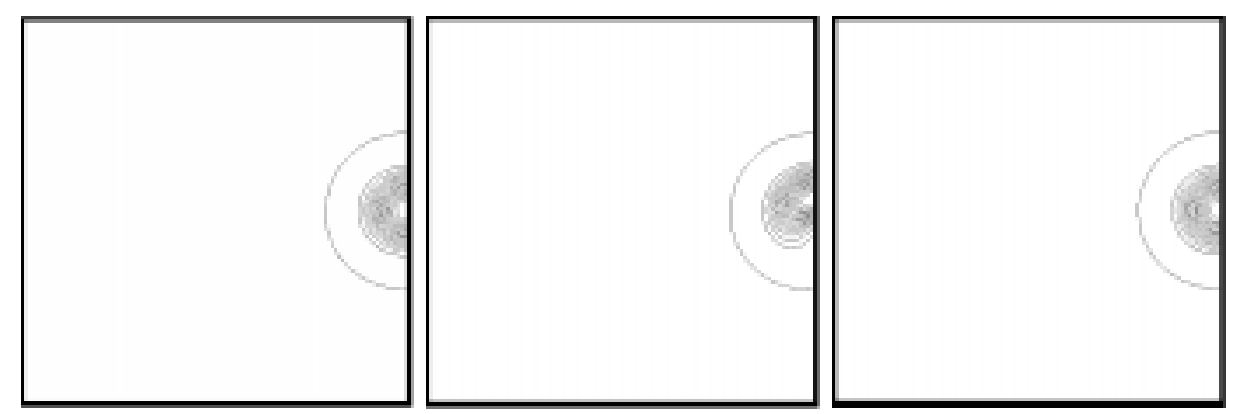

vorticity isolines
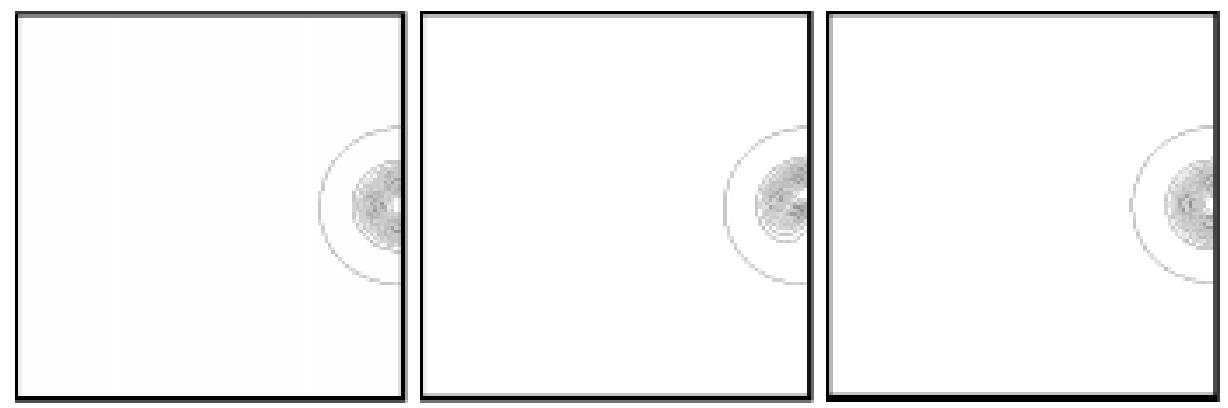

pressure isolines

Figure 6. Comparison of the NRBC solution (middle) and the PBC solution (right) to the reference solution (left).

The second test case corresponds to the flow over a $2 D$ ramp with an angle $\beta=10^{\circ}$ and the computational domain $\Omega$ is taken in the neighbourhood of the corner as illustrated in Figure 5. The inflow condition is a Blasius flow developed from the beginning of the plate which is equal to $(1,0)^{t}$ far from the boundary layer. Here, the reference flow $U^{\text {ref }}$ is taken equal to the steady flow computed on a larger domain with the technique of the buffer zone at $R e=50$ and the convection velocity is taken equal to $(1,0)^{t}$ (see [5] for more details). The first run is to compute the solution at the same Reynolds number on the domain $\Omega$ applying our boundary condition using the flow computed with the buffer zone as initial datum. Then, the solution remains stable without any changes and keeps positive velocities in the whole domain. The second run is to increase the Reynolds number to $R e=100$. After a while, a new steady solution with a recirculation zone behind the corner is obtained like with the buffer zone technique whereas the NRBC provides a periodic solution. The last run is to increase again the Reynolds number to $R e=400$ taking the steady solution at $R e=100$ as reference flow. This time a more complex solution with strong vortices coming from the corner is obtained. These vortices grow as they are convected along the ramp and cross the artificial boundary without any visible reflections. The solution after a long time of simulation is close to the one obtained with the buffer zone technique despite a small delay (Fig. 7). A Fourier analysis of the time trace recorded at some points located behind the corner show that the same frequencies appear in the two solutions whereas the spectrum of the NRBC solution is dominated by a low frequency already present at $R e=100$ which corresponds to the travel of acoustic waves from the artificial boundary back to the corner (see [5] for more details). 


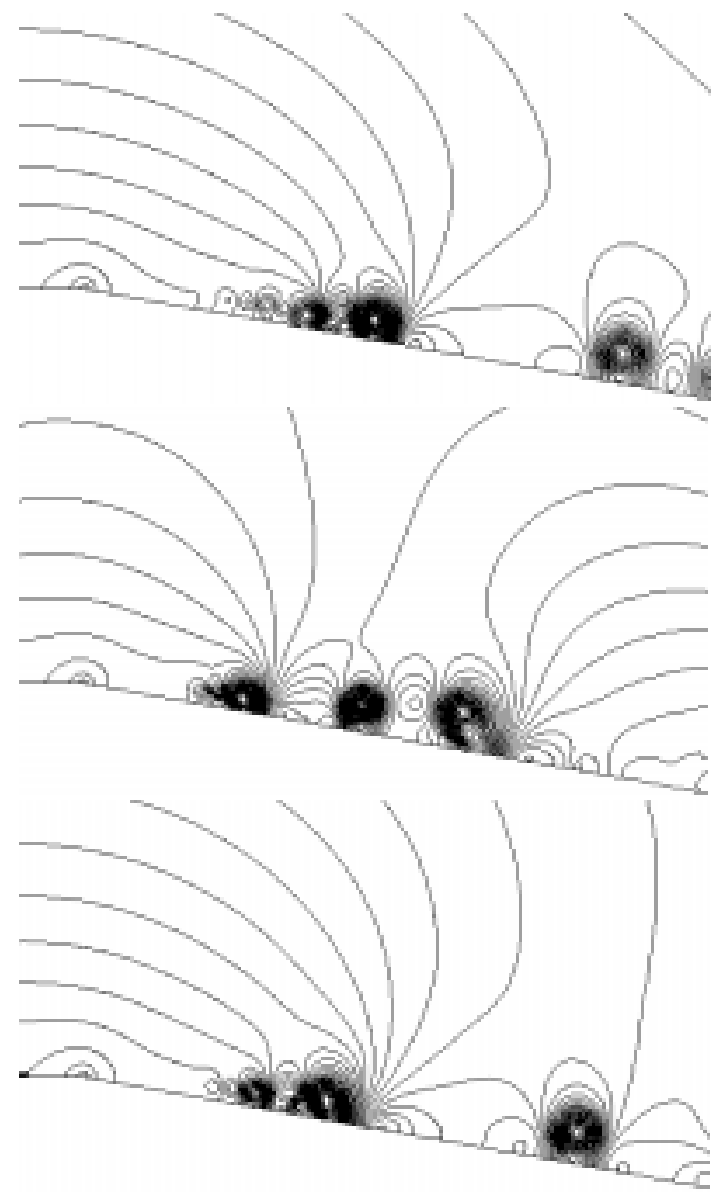

pressure isolines

Figure 7. Comparison of the NRBC solution (middle) and the PBC solution (bottom) to the reference solution (top).

\section{Conclusions}

In this paper, we show that the introduction of a reference flow is a very efficient way to derive non reflecting boundary conditions. For incompressible flows a Neumann-type boundary condition coupled to the traction of a reference flow yields a nearly transparent boundary condition. For compressible flows the characteristic waves amplitudes of the Euler part of the equations are discretized using a reference flow and a convection velocity to avoid reflections of acoustic waves. In both cases, a direct numerical simulation of complex flows can be achieved without the help of the buffer zone technique which is not completely handled and induces a significant increase of the computing time.

\section{REFERENCES}

[1] Ph. Angot, Ch.-H. Bruneau and P. Fabrie, A penalization method to take into account obstacles in incompressible viscous flows. Numer. Math. 81 (1999).

[2] E. Arquis and J. P. Caltagirone, Sur les conditions hydrodynamiques au voisinage d'une interface milieu fluide - milieu poreux: application à la convection naturelle. C. R. Acad. Sci. Paris 299, Série II (1984). 
[3] Ch.-H. Bruneau, Numerical Simulation and Analysis of the Transition to Turbulence. 15th ICNMFD, Lect. Notes in Phys. 490 (1996).

[4] Ch.-H. Bruneau, Numerical Simulation of incompressible flows and analysis of the solutions. CFD Review Vol. I (1998).

[5] Ch.-H. Bruneau and E. Creusé, Towards a transparent boundary condition for compressible Navier-Stokes equations (submitted).

[6] Ch.-H. Bruneau and P. Fabrie, Effective downstream boundary conditions for incompressible Navier-Stokes equations. Int. J. Numer. Methods in Fluids 19 (1994).

[7] Ch.-H. Bruneau and P. Fabrie, New efficient boundary conditions for incompressible Navier-Stokes equations: a well-posedness result. Mod. Math. Anal. Num. 30 (1996).

[8] Ch.-H. Bruneau, O. Greffier and H. Kellay, Numerical study of grid turbulence in two dimensions and comparison with experiments on turbulent soap films. Phys. Rev. E 60, No. 2, (1999).

[9] J.P. Caltagirone, Sur l'interaction fluide-milieu poreux: application au calcul des efforts exercés sur un obstacle par un fluide visqueux. C.R. Acad. Sci. Paris 318, Série II, (1994).

[10] J.R. Chasnov, The viscous-convective subrange in nonstationary turbulence. Phys. Fluids 10, No. 5, (1998).

[11] T. Colonius, S.K. Lele and M. Parviz, Boundary conditions for direct computation of aerodynamic sound generation. AIAA journal 31 (1993).

[12] B. Enquist and A. Majda, Absorbing boundary conditions for the numerical simulation of waves. Math. Comp. 31 (1977).

[13] P.M. Gresho, Incompressible fluid dynamics: some fundamental formulation issues. Ann. Rev. Fluid Mech. 23 (1991).

[14] H. Kellay, Ch.-H. Bruneau, A. Belmonte and X. L. Wu, Probability density functions of the enstrophy flux in two dimensional grid turbulence. Phys. Rev. Lett. (to appear).

[15] H. Kellay, X.L. Wu and W. I. Goldburg, Experiments with turbulent soap films. Phys. Rev. Lett. 74 (1995).

[16] H. Kellay, X.L. Wu and W.I. Goldburg, Vorticity measurements in turbulent soap films. Phys. Rev. Lett. 80 (1998).

[17] H.O. Kreiss, Initial boundary value problems for hyperbolic systems. Comm. P. App. Math. 23 (1970).

[18] M. Marion and R. Temam, Navier-Stokes equations: theory and approximation. Handbook of numerical analysis, Vol. VI, (1998).

[19] T.J. Poinsot and S.K. Lele, Boundary conditions for direct simulations of compressible viscous flows. J. Comp. Phys. 101 (1992).

[20] D.H. Rudy and J.C. Strikwerda, A nonreflecting outflow boundary condition for subsonic Navier-Stokes calculations. J. Comp. Phys. 36 (1980).

[21] J.C. Strikwerda, Initial boundary value problems for incompletely parabolic systems. Comm. P. App. Math. 30 (1977).

[22] R. Temam, Navier-Stokes equations and numerical analysis. North-Holland (1979).

[23] B. Wasistho, B.J. Geurts and J.G.M. Kuerten, Simulation techniques for spatially evolving instabilities in compressible flows over a flat plate. Computers and Fluids 26 (1997).

[24] C.H. Williamson, Oblique and parallel modes of vortex shedding in the wake of a circular cylinder at low Reynolds numbers. J. Fluid Mech. 206 (1989). 